 \\ REVISTA PREVENCÃO DE INFECCÃO E SAÚDE \\ (REPIS)
}

\section{Custos da antibioticoterapia em pacientes adultos com infecção hospitalar em uma unidade de}

\section{terapia intensive}

Costos de la antibioticoterapia en pacientes adultos con infección hospitalaria en una unidad de terapia intensiva

Costs of antibiotic therapy in adult patients with hospital infection in an intensive care unit

Fernanda Letícia Oliveira Lara ${ }^{1}$, Arthur Velloso Antunes ${ }^{1}$, Clesnan Mendes-Rodrigues ${ }^{2}$, Igor Oliveira Felice ${ }^{3}$

1. Faculdade de Medicina, Universidade Federal de Uberlândia, Uberlândia, Brasil.

2. Instituto de Biologia, Estatística, Faculdade de Matemática, Universidade Federal de Uberlândia, Uberlândia, Brasil.

3. Faculdade de Engenharia Mecânica, Universidade Federal de Uberlândia, Uberlândia, Brasil.

\begin{abstract}
Objective: to evaluate hospital infection rates $(\mathrm{IH})$ and costs with antibiotic therapy in adult patients with $\mathrm{HI}$ of an intensive care unit of a university hospital. Method: This is a retrospective exploratory descriptive study, carried out at the Adult Intensive Care Unit of the Hospital de Clínicas de Uberlândia. The number of IHs, antibiotics in use, time of use and cost were collected. Results: Of 1119 patients, $328(29.30 \%)$ had IH, with a high HI level of $44.71 \mathrm{IH}$ per 1000 patient-days. The cost of antimicrobials was USD496851.90 per year, $25 \%$ of the cost of hospital medicines. The average cost per patient was USD1514.79. Twenty-seven antimicrobials were used, of which cefepime, teicoplanin, vancomycin and meropenem were the most used, while the most expensive were tigecycline, piperacicline, ertapenen and polymyxin B (57.8\% of costs). Conclusion: The results evidenced the need to create a policy for the use and rationalization of antimicrobials.
\end{abstract}

Key-Words: Costs and Cost Analysis; Anti-Bacterial Agents; Cross Infection; Intensive Care Units.

\section{RESUMO}

Objetivo: avaliar as taxas de infecção hospitalar (IH), os custos com antibioticoterapia em pacientes adultos com IH de uma unidade terapia intensiva de um hospital universitário. Método: Estudo descritivo exploratório retrospectivo, realizado na Unidade de Terapia Intensiva de Adultos do Hospital de Clínicas de Uberlândia. Coletou-se o número de IHs, os antibióticos em uso, tempo de uso e custo. Resultados: De 1119 pacientes $328(29.30 \%)$ apresentavam IH, com taxa de IH alta de $44,71 \mathrm{IH}$ por 1000 paciente-diaO custo de antimicrobianos foi USD496851,90 por ano, 25\% do custo de medicamentos do hospital. 0 custo médio por paciente foi de USD1514,79. Foram utilizados 27 antimicrobianos, sendo cefepime, teicoplanina, vancomicina e meropenem os mais usados, enquanto os mais caros eram tigeciclina, oiperaciclina, ertapenen e polimixina B ( $57,8 \%$ dos custos). Conclusão: Os resultados evidenciaram a necessidade da criação de uma política de uso e racionalização de antimicrobianos.

Palavras-chave: Custo; antimicrobianos; antibacterianos; infecção hospitalar; unidade de terapia intensiva.

\section{RESUMÉN}

Objetivo: evaluar las tasas de infección hospitalaria $(\mathrm{IH})$, los costos con antibioticoterapia en pacientes adultos con $\mathrm{IH}$ de una unidad de cuidados intensivos de un hospital universitario. Método: Estudio descriptivo exploratorio retrospectivo, realizado en la Unidad de Terapia Intensiva de Adultos del Hospital de Clínicas de Uberlândia. Se recogió el número de IHs, los antibióticos en uso, tiempo de uso y costo. Resultados: De 1119 pacientes 328 (29.30\%) presentaban $\mathrm{IH}$, con una tasa de $\mathrm{IH}$ alta de $44,71 \mathrm{IH}$ por 1000 paciente-día. El costo de los antimicrobianos fue de USD 496851,90 por año, el $25 \%$ del costo de los medicamentos del hospital. El costo promedio por paciente fue de USD1514,79. Se utilizaron 27 antimicrobianos, siendo cefepime, teicoplanina, vancomicina y meropenem los más usados, mientras que los más caros eran tigeciclina, oiperaciclina, ertapenen y polimixina B ( $57,8 \%$ de los costos). Conclusión: Los resultados evidenciaron la necesidad de crear una política de uso y racionalización de antimicrobianos. Descriptores: Costos y Análisis de Costo; antibacterianos; Infección Hospitalaria; Unidades de Cuidados Intensivos.

\section{Como citar este artigo:}

Lara FLO, Antunes AV, Mendes-Rodrigues C, Felice IO. Custos da antibioticoterapia em pacientes adultos com infecção hospitalar em uma unidade de terapia intensive. Rev Pre Infec e Saúde[Internet].2017;3(4):814. Available from: http://www.ojs.ufpi.br/index.php/nupcis/article/view/6621 


\section{INTRODUÇÃO}

A infecção hospitalar (IH) é qualquer infecção adquirida após a internação do paciente e que se manifeste durante a internação, ou mesmo após a alta, quando puder ser relacionada com a internação ou procedimentos hospitalares. ${ }^{(1-3)}$ Os pacientes provenientes de outro hospital que se internam com infecção são considerados portadores de IH do hospital de origem e devem ser notificados. ${ }^{(3)}$

As Infecções Hospitalares ou infecções relacionadas a assistência a saúde (IRAS) são as complicações mais frequentes e mais sérias em Unidades de Terapia Intensiva (UTI). Os índices de infecção hospitalar em UTI apresentam porcentagem mais elevadas quando comparado com as outras unidades de internação. ${ }^{(4)}$ Já que neste ambiente 0 paciente encontra-se mais exposto ao risco de infecção, por sua condição clínica e variedade de procedimentos invasivos realizados rotineiramente. 0 risco de infecção é diretamente proporcional à gravidade da doença, as condições nutricionais, a natureza dos procedimentos diagnósticos e ou terapêuticos, bem como, ao tempo de internação, dentre outros aspectos. ${ }^{(3 ; 5-7)}$

As infecções hospitalares ocasionam custos consideráveis aos sistemas de saúde de todo o mundo, por isso o uso inadequado de antimicrobiano, o aumento dos dias de internação e os consequentes custos com terapêutica antimicrobiana, além das questões pessoais, como alterações psicológicas dos pacientes, evidenciam a importância da prevenção e controle das infecções hospitalares. Uma das medidas mais importantes é o controle do uso dos antibióticos e consequentemente $\mathrm{o}$ controle da resistência bacteriana.

As principais consequências da resistência bacteriana são: o aumento do custo e do tempo de tratamento, pela utilização de medicamentos mais caros e até mais tóxicos; aumento do tempo de hospitalização; isolamento do paciente; aumento da frequência e da gravidade das infecções hospitalares; aumento da taxa de mortalidade associada a este tipo de infecção. ${ }^{(7-10)}$

O impacto econômico da resistência bacteriana afeta de forma diferente os diversos atores envolvidos no processo de fornecimento e uso de antimicrobianos. O prescritor tem o custo da ineficácia da terapia convencional, com eventual perda de pacientes. 0 paciente tem o custo da doença não solucionada e de eventual morte, onerando-se com a exigência de medicamento alternativo. Por sua vez, o Sistema Público de Saúde gasta excessivamente, desequilibrando recursos geralmente escassos. ${ }^{(11)} \mathrm{A}$ problemática da IH no Brasil cresce a cada dia, considerando que o custo do tratamento dos clientes com IH é três vezes maior que o custo dos clientes sem infecção. Mesmo com a legislação vigente no país, os índices de IH permanecem altos e com um agravante, o fato das instituições de saúde pública possuírem as maiores taxas de prevalência de IH no país.

Assim, o objetivo deste estudo foi analisar as taxas de infecções associadas a assistência a saúde, o perfil de uso de antibióticos e o custo com antibioticoterapia em pacientes com Infecção Hospitalar, internados na Unidade de Terapia Intensiva (UTI) de Adultos de um Hospital Universitário.

\section{MÉTODOS}

Trata-se de um estudo descritivo exploratório retrospectivo, realizado na Unidade de Terapia Intensiva de Adultos do Hospital de Clínicas de Uberlândia da Universidade Federal de Uberlândia. O Hospital de Clínicas de Uberlândia (HCU-UFU) é um hospital público de alta complexidade e referência para uma população de mais de dois milhões de habitantes. Localizado na região do Triângulo Mineiro, estado de Minas Gerais, oferece atendimentos de urgência e emergência, ambulatorial, cirúrgico e internação. 
Lara FLO et al

O estudo foi realizado na UTI de Adultos deste Hospital que é composta por 30 leitos de terapia intensiva, sem regulação por complexidade ou especialidade. A taxa de ocupação é de $100 \%$ com intervalos médios de três horas de desocupação entre altas e admissões.

Os dados referentes aos pacientes que foram acometidos com IH foram coletados nos bancos de dados do Serviço de Controle de Infecção Hospitalar (SCIH) do HC-UFU, nas Fichas de Vigilância Epidemiológica da UTI de Adultos, em que são feitos os registros dos dados dos pacientes através de busca ativa para levantamento dos casos de $\mathrm{IH}$. Os dados referentes aos antimicrobianos administrados aos pacientes acometidos com IH no período da coleta de dados foram coletados nos prontuários destes pacientes. Os dados referentes aos custos dos antimicrobianos utilizados foram coletados junto ao setor de compras da instituição.
Infecções hospitalares em UTI

Os custos foram convertidos em dólares, em cotação do período. Foram incluídos na pesquisa 1119 pacientes internados durante um ano 2013.

O projeto foi aprovado no Comitê de Ética em Pesquisa da Universidade Federal de Uberlândia com a liberação do uso do Termo de Consentimento Livre e Esclarecido pelo Parecer 862.832 e seguiu as normas previstas na Resolução 466/2012 do Conselho Nacional de Saúde.

\section{RESULTADOS}

A percentagem de doentes com IRAS foi de 29,30\%. Em muitos casos, os pacientes foram afetados por mais de uma IRAS, resultando em um total de 483 episódios de IRAS, resultando em uma taxa de IRAS de 44,71 IRAS por 1000 paciente-dia, oscilando mensalmente de 30,19 a 61,82 IRAS por 1000 paciente-dia (Tabela 1$)$.

Tabela 1 - Distribuição mensal do número de pacientes internados na UTI, pacientes que foram acometidos com infecção relacionada a assistência a saúde (IRAS), porcentagem de pacientes com IRAS, número de IRAS e taxa de IRAS em uma Unidade de Terapia Intensiva de um hospital universitário, 2013.

\begin{tabular}{cccccc}
\hline Mês & $\begin{array}{c}\text { Pacientes } \\
\text { internados }\end{array}$ & $\begin{array}{c}\text { Pacientes com } \\
\text { IRAS }\end{array}$ & $\begin{array}{c}\text { Percentagem de } \\
\text { Pacientes com } \\
\text { IRAS }\end{array}$ & $n^{\circ}$ de IRAS & $\begin{array}{c}\text { Taxa de IRAS } \\
\text { (IRAS por 1000 } \\
\text { paciente-dia) }\end{array}$ \\
\hline Janeiro & 85 & 19 & 22,35 & 29 & 32,22 \\
Fevereiro & 80 & 18 & 23,70 & 25 & 30,19 \\
Março & 104 & 35 & 33,60 & 44 & 47,83 \\
Abril & 88 & 32 & 36,30 & 43 & 47,94 \\
Maio & 91 & 29 & 31,80 & 42 & 45,90 \\
Junho & 86 & 29 & 33,70 & 43 & 48,64 \\
Julho & 103 & 33 & 32,00 & 48 & 52,52 \\
Agosto & 98 & 24 & 24,40 & 38 & 41,21 \\
Setembro & 88 & 26 & 29,50 & 43 & 48,31 \\
Outubro & 101 & 28 & 27,70 & 41 & 44,57 \\
Novembro & 102 & 20 & 19,60 & 30 & 33,67 \\
Dezembro & 93 & 35 & 37,60 & 57 & 61,82 \\
\hline Total & 1119 & 328 & 29,30 & 483 & 44,71 \\
\hline
\end{tabular}

Foram utilizados 27 antimicrobianos diferentes nos 328 pacientes com IRAS (Tabela 2). É importante enfatizar que os antibióticos tópicos foram excluídos do estudo devido à dificuldade em 
Lara FLO et al

dimensionar a quantidade administrada ao paciente e em muitas vezes uma unidade é usada por mais de um paciente ao mesmo tempo. 0 número de pacientes que usaram cada antimicrobiano, o custo total, o custo médio por paciente, a mediana de dias de uso e o custo
Infecções hospitalares em UTI

médio por paciente por dia apresentaram grande oscilação. Os antibióticos mais utilizados foram Cefepime, Teicoplanina, Vancomicina e Meropenem. Enquanto os antibióticos menos utilizados foram Cefuroxima, Aciclovir, Anfotericina B e Gentamicina. (Tabela 2)

Tabela 2 - Antibióticos utilizados nos pacientes acometidos com infecções relacionadas a assistência a saúde e internados na Unidade de Terapia Intensiva de Adultos de um hospital universitário, custo total, custo médio por paciente, tempo mediano de uso e custo médio por paciente por dia de internação. Uberlândia 2013.

\begin{tabular}{|c|c|c|c|c|c|}
\hline Antibiótico & $\begin{array}{c}\mathrm{n}^{\circ} \text { de } \\
\text { pacientes (\%) } \\
n=328\end{array}$ & $\begin{array}{l}\text { Custo total } \\
\text { (USD\$) }\end{array}$ & $\begin{array}{l}\text { Custo médio } \\
\text { por paciente } \\
\text { (USD\$) }\end{array}$ & $\begin{array}{c}\text { Tempo } \\
\text { mediano de } \\
\text { uso (dia) }\end{array}$ & $\begin{array}{l}\text { Custo médio por } \\
\text { paciente por dia } \\
\text { (USD\$) }\end{array}$ \\
\hline Aciclovir & $2(0,6)$ & 635,36 & 317,68 & 12,5 & 25,41 \\
\hline Amicacina & $9(2,7)$ & 53,91 & 5,99 & 14 & 0,43 \\
\hline Ampilicina & $30(9,1)$ & 295,86 & 9,86 & 12 & 0,82 \\
\hline Ampicilina + Sulbactam & $17(5,2)$ & 904,28 & 53,19 & 11 & 4,83 \\
\hline Anfotericina B & $2(0,6)$ & 1200,14 & 600,07 & 9,5 & 63,16 \\
\hline Cefazolina & $18(5,5)$ & 158,31 & 8,80 & 4 & 2,20 \\
\hline Cefepima & $190(57,9)$ & 13593,30 & 71,54 & 9 & 7,95 \\
\hline Ceftriaxona & $74(22,6)$ & 705,37 & 9,53 & 8 & 1,19 \\
\hline Cefuroxima & $1(0,3)$ & 3,28 & 3,28 & 2 & 1,64 \\
\hline Ciprofloxacina & $71(21,6)$ & 1751,28 & 24,66 & 9 & 2,74 \\
\hline Clindamicina & $67(20,4)$ & 821,28 & 12,26 & 9 & 1,36 \\
\hline Daptomicina & $7(2,1)$ & 18232,96 & 2604,71 & 10 & 260,47 \\
\hline Erpapenem & $70(21,3)$ & 81261,51 & 1160,88 & 9 & 128,99 \\
\hline Gentamicina & $3(0,9)$ & 7,79 & 2,59 & 8 & 0,33 \\
\hline Imipenem & $45(13,7)$ & 6748,91 & 149,98 & 11 & 13,63 \\
\hline Levofloxacina & $22(6,7)$ & 833,71 & 37,90 & 10 & 3,79 \\
\hline Meropenem & $115(35)$ & 26470,99 & 230,18 & 12 & 19,18 \\
\hline Metronidazol & $31(9,5)$ & 391,58 & 12,63 & 9 & 1,41 \\
\hline Micafugina & $26(7,9)$ & 27807,11 & $1069 ; 50$ & 9 & 118,83 \\
\hline Moxifloxa & $8(2,4)$ & 5230,90 & 653,86 & 10 & 65,39 \\
\hline Oxacilina & $35(10,7)$ & 1971,05 & 56,32 & 9 & 6,26 \\
\hline Piperaciclina & $71(21,6)$ & 87858,01 & 1237,44 & 9 & 137,49 \\
\hline Polimixina B & $67(20,4)$ & 60107,73 & 897,13 & 11 & 81,56 \\
\hline $\begin{array}{c}\text { Sulfametoxazol + } \\
\text { trimetoprima }\end{array}$ & $23(7)$ & 2518,87 & 109,52 & 10 & 10,95 \\
\hline Teicoplanina & $138(42)$ & 34957,93 & 253,32 & 11 & 23,03 \\
\hline Tigeciclina & $42(12,8)$ & 117865,52 & 2806,32 & 11 & 255,12 \\
\hline Vancomicina & $126(38,4)$ & 4464,97 & 35,44 & 11 & 3,22 \\
\hline Total & 328 & 496851,90 & 1514,79 & 9,6 & 157,79 \\
\hline
\end{tabular}


Lara FLO et al

\section{DISCUSSÃO}

Em um estudo de prevalência de um dia realizado em dezesseis UTIs de diferentes municípios do Rio Grande do Sul ${ }^{(12)}$, 71\% dos pacientes apresentavam IRAS. Outro hospital universitário do município de Teresina, no Estado do Piauí (2), observou que na UTI Geral há uma taxa de 64 IRAS por 1000 paciente-dia, enquanto que na UTI de Emergência foi de 36 IRAS por 1000 paciente-dia. Essas UTIs têm perfil semelhante a UTI estudada aqui. Apesar das semelhanças entre as unidades, os níveis de IRAS foram muito mais elevados do que aqueles encontrados por nós 44,71 IRAS 1000 paciente-dia. As taxas de prevalência de IRAS para pacientes com UTI variam de acordo com o tipo de unidade e a população, doença e perfil do paciente atendidos (2). As taxas de IRAS nas UTI variam de 18 a 54 IRAS por 1000 paciente-dia, sendo cerca de cinco vezes maior que outras unidades de internação(13). No entanto, outros estudos encontraram taxas de IRAS realmente inferiores às apresentadas neste estudo; Oscilando de 15,95 a 20 IRAS por 1000 pacientedia ${ }^{(14)}$.

Em um estudo descritivo sobre o padrão de consumo antibacteriano em uma UTI geral de um hospital regional o consumo de mais de um tipo de antibacteriano foi freqüente, com uma média de 2,54 e uma mediana de 2 tipos antibacterianos por paciente ${ }^{(15)}$. Um paciente da amostra utilizou nove tipos diferentes de antibacterianos durante a estadia $^{(15)}$. Neste estudo presente, dos 328 pacientes com IH que usaram antibacterianos, apenas 10 foram restritos a 1 tipo de antimicrobianos e dentro desta amostra havia pacientes que usaram até 9 tipos diferentes de antibacterianos durante a sua internação.

Os dados de custo dos antimicrobianos demonstraram que o uso desses é altamente dispendioso para a instituição e para o sistema de saúde e que seu uso deve ser controlado e liberado apenas nos casos extremamente necessários. Os quatro antibióticos mais caros utilizados,
Infecções hospitalares em UTI

Tigeciclina, Piperacicline, Ertapenem e Polimixina B, correspondem a um custo de USD\$347.092,77, equivalente a $57,8 \%$ do custo total com antibiótico no ano. Tais antimicrobianos, foram utilizados em um grande número de pacientes, embora com seu alto custo, o que pode demonstrar a necessidade de maior controle de uso.

Existem poucos estudos comparativos de custo com antimicrobianos. De acordo com um estudo feito em uma UTI das Forcas Armadas, um custo total de USD\$18,519.41 com antibióticos ocorreu $^{(16)}$. Este valor foi dividido quase igualmente entre os $90 \%$ dos pacientes que receberam até três antibióticos sucessivamente e $10 \%$ que receberam mais de três e até seis antibióticos ${ }^{(16)}$. Um custo médio de USD\$ 874,50 dólares foi encontrado para uma UTI de um hospital em Belo Horizonte ${ }^{(17)}$. Esses valores são bem inferiores aos observados por nosso estudo.

Esses resultados sugerem a necessidade e a importância de uma política de controle para prescrever esses medicamentos, com a institucionalização de protocolos de redução de custos e até mesmo as conseqüências do alto consumo de antimicrobianos, associado ao aumento de microorganismos resistentes, afetando não apenas o paciente com infecção, mas também a instituição e o sistema de saúde para aumentar o tempo de hospitalização e os custos de tratamento.

\section{REFERÊNCIAS}

1. Brasil. Ministério da Saúde. Portaria n. 2.616, 12 de maio de 1998. Diário Oficial da União, Brasília, 13 de maio de 1998. Seção 1, p. 133. Acesso em 01 de Dezembro de 2017. Available from:

http://bvsms.saude.gov.br/bvs/saudelegis/gm/199 8/prt2616_12_05_1998.html

2. Moura MEB, Campelo SMA, Brito FCP, Batista OMA, Araujo TME, Oliveira ADS. Infecção hospitalar: estudo de prevalência em um hospital 
Lara FLO et al

público de ensino. Rev. Bras. de Enferm. 2007 julho/agosto; 13(4): 416- 421. Available from: http://www.scielo.br/pdf/reben/v60n4/a11.pdf

3. Padrão MC, Monteiro ML, Maciel NR, Viana FFCF, Freitas NA. Prevalência de infecções hospitalares em unidade de terapia intensiva. Rev. Bras. Clín. Méd.. 2010; 8(2): 125-128. Available from: $\quad$ http: //files.bvs.br/upload/S/16791010/2010/v8n2/a007.pdf

4. Lichy RF, Marques IR. Fatores de risco para infecção hospitalar em unidades de terapia intensiva: atualização e implicações para a enfermagem. Rev. Enf. Univ. St Amaro. 2002; 3(1):43-49. Available

from:

http://unifia.edu.br/revista_eletronica/revistas/sa ude_foco/artigos/ano2016/080_fatores_de_risco_e _prevencao_para_infeccao_hospitalar.pdf

5. Andrade D, Leopoldo VC, Hass V J. Ocorrência de bactérias multirresistentes em um Centro de Terapia Intensiva de Hospital Brasileiro de Emergências. Rev. Bras. Ter. Intensiva 2006 janeiro/março; 8(1): 27-33. Available from: http://www.scielo.br/pdf/rbti/v18n1/a06v18n1.pd $f$

6. Jacoby TS. Associação entre consumo de antimicrobianos e multirresistência bacteriana em centro de terapia intensiva de hospital universitário brasileiro, 2004-2006. [Dissertação]. Porto Alegre: Universidade Federal do Rio Grande do Sul. Faculdade de Medicina. Programa de PósGraduação em Medicina: Ciências. Médicas; 2008. Available

from: http: / / www.lume.ufrgs.br/bitstream/handle/1018 3/13066/000638076.pdf?sequence $=1$

7. Fernandes IQ, Sousa HF, Brito MAM, Tavares SN, Matos VC, Sousa MO. Impacto farmacoeconômico da racionalização do uso de antimicrobianos em unidades de terapia intensiva.

Rev. Bras. Farm. Hosp. Serv. Saúde. 2012 outubro/novembro; 3(4): 10-14. Available from: http://www.sbrafh.org.br/rbfhss/public/artigos/2 012030417BR.pdf
Infecções hospitalares em UTI

8. Gurgel TC, Carvalho WSA. Assistência farmacêutica e o aumento da resistência bacteriana aos antimicrobianos. Lat. Am. J. Pharm. 2008; 27(1): 118-123. Available from: http://www.latamjpharm.org/resumenes/27/1/LA JOP_27_1_7_1.pdf

9. Lima ME, Andrade D, Hass VJ. Avaliação prospectiva da ocorrência de infecção em pacientes críticos de unidade de terapia intensiva. Rev. Bras. Ter. Intensiva. 2007; 19(3): 342- 347. Available from: http://www.scielo.br/pdf/rbti/v19n3/v19n3a13.pd $f$

10. Paula AO. Custos com antimicrobianos no tratamento de pacientes com infecção da corrente sanguínea em uma Unidade de Terapia Intensiva. [Dissertação]. Belo Horizonte: Escola de Enfermagem, Universidade Federal de Minas Gerais; 2011. Available from: http://www.enf.ufmg.br/pos/defesas/707M.PDF

11. Rodrigues FD, Bertoldi AD. Perfil da utilização de antimicrobianos em um hospital privado. Ciênc. Saúde Coletiva. 2010; 15(Supl.1): 1239-1247. Available from: http://www.scielo.br/pdf/csc/v15s1/033.pdf

12. Lisboa T, Faria M, Hoher JA, Borges LAA, Gômes J, Schifelbaln L, et al. Prevalência de infecção nosocomial em unidades de terapia intensiva do Rio Grande do Sul. Rev. Bras. Ter. Intensiva. 2007 outubro/dezembro; 19(4), 414420. Available from: http://www.scielo.br/pdf/rbti/v19n4/a02v19n4.pd $f$

13. Oliveira AC, Kovner CT, Silva RS. Infecção hospitalar em unidade de tratamento intensivo de um hospital universitário brasileiro. Rev. LatinoAm. Enfermagem. 2010 março/abril; 18(2): 96-104. Available

from: http://www.scielo.br/pdf/rlae/v18n2/pt_14.pdf 14. Leiser JJ, Tognim MCB, Bedendo J. Infecções hospitalares em um centro de terapia intensiva de um Hospital de ensino no norte do Paraná. Ciênc. Cuid. Saúde. 2007 abril/junho; 6(2): 
Lara FLO et al

$181-186$

Available

from:

http://www. periodicos.uem.br/ojs/index.php/Cie ncCuidSaude/article/view/4149/2730

15. Carneiro JCO. Padrão de consumo de antibacterianos em uma UTI geral: correlação com a resistência bacteriana. Faculdade de Medicina, Universidade de Brasília, 2006. Available from: http://www.repositorio.unb.br/bitstream/10482/5 221/1/Dissert\%20Julio\%20Cesar\%20de\%200liveira\%2 oCarneiro.pdf

\section{AGRADECIMENTOS}

Aos profissionais que atuam no Serviço de Controle de Infecção Hospitalar do Hospital de Clínicas de Uberlândia pela presteza e colaboração no momento da coleta de dados.

\section{COLABORAÇÕES}

Os autores AVA, CMR e FLOL participaram de forma conjunta das etapas de concepção e delineamento do estudo, análise e interpretação dos dados. AVA, FLOL CMR e IOF escreveram e fizeram a revisão crítica do conteúdo intelectual do manuscrito, aprovação final da versão a ser publicada e responsabilidade por todos os aspectos do trabalho, incluindo a garantia de sua precisão e integridade.

\section{CONFLITOS DE INTERESSE}

Não há conflitos de interesse a declarar

\section{CORRESPONDENCIA}

Arthur Velloso Antunes

Campus Umuarama - Bloco 2U - Sala 23, Av. Pará, 1720 - Bairro Umuarama

Uberlândia - MG - CEP 38400-902

E-mail: arthurantunes22@gmail.com intensiva: Un esfuerzo racional? Rev. Serv. Sanidad de las Fuerzas Armadas. 1985. 11, 17-20,

17. Oliveira AC de, Oliveira PA de. Discontinuation of antimicrobials and costs of treating patients with infection. Acta Paul. Enferm. 2012 Mar; 25 (spe2): 68-74. Available from: http://www.scielo.br/pdf/ape/v25nspe2/11.pdf 